the number of the men with documented as primary, secondary, or early latent syphilis (PSELS) by ICD_9 codes.

Results Of 52,771 MSM, 33.8\% were aged 15-29 years, $89.6 \%$ had private insurance, $51.0 \%$ resided in South, and $74.8 \%$ had tests ordered by infectious disease specialists. $14.5 \%$ had no syphilis tests, $4.8 \%$ had TT only $(38.2 \%$ were reactive), $63.0 \%$ had NTT only $(2.0 \%$ were reactive), and $17.7 \%$ had both NTT and TT $(8.3 \%$ neither were reactive and $86.4 \%$ both were reactive). Of 45,108 MSM who had syphilis tests, $1.1 \%$ had PSELS. Of 2,547 MSM who had TT only, the number of tests in the two years was one $(57.1 \%)$, two $(22.3 \%)$, and three (13.3\%). Of 33,238 MSM who had NTT only, the number of tests was one (53.5\%), two (23.3\%), and three (11.1\%). Of 9323 MSM who had both NTT and TT, the number of tests was one (30.4\%), two (24.7\%), three $(17.8 \%)$, and four (12.8\%). Of 11870 men who had reactive TT, $51.8 \%$ had $\geq$ two reactive TT. CT and GC were significantly higher in men with reactive vs. nonreactive NTT and TT: rectal CT $(24.4 \%$ vs. $12.6 \%)$ or GC (19.8\% vs. $9.9 \%)$; pharyngeal CT $(5.4 \%$ vs. $2.9 \%)$ or GC $(14.7 \%$ vs. $10.6 \%)$; urethral CT $(7.6 \%$ vs. $5.7 \%)$ or GC (8.0\% vs. $5.3 \%)$.

Conclusion Syphilis testing was common among men who were tested for rectal CT or GC, but most were tested only once. Men with reactive syphilis tests were more likely to have GC or CT vs. men with nonreactive tests. Routine and timely syphilis testing should be prioritised.

\section{P3.89 PREVALENCE, TREND, OUTCOMES AND RISK FACTORS FOR LATE PRESENTATION FOR HIV CARE IN ETHIOPIA, 2003-2015}

${ }^{1}$ Hailay Gesesew, ${ }^{1}$ Lillian Mwanri, ${ }^{1}$ Paul Ward, ${ }^{2}$ Kifle Hajito. ${ }^{1}$ Flinders University, Adelide, Australia; ${ }^{2}$ Jimma University, Jimma, Ethiopia

\subsection{6/sextrans-2017-053264.324}

Introduction Late presentation for HIV care (LP) delays the achievements of the 90-90-90 UNAIDS target, a program in which Ethiopia has subscribed for. However, the prevalence, trend, outcomes and risk factors of LP among children and adults were not assessed very well in the nation.

Methods 12 years retrospective cohort study was conducted using data extracted from an antiretroviral therapy (ART) clinic in Southwest Ethiopia. LP for children and adults was measured using CD4 lymphocyte counts and WHO clinical stages. We described the percentage of LP by mortality, discontinuation from ART and immunological failure to show outcomes of LP. The analysis of descriptive and inferential statistics (logistic regression) was undertaken. Missing data were handled using multiple imputations assuming missing at random (MAR) pattern.

Results Of the 8172 patients enrolled for HIV care between June 2003 and March 2015, 5299 (64.8\%) patients were on ART: 4900 (92.5\%) were adults and 399 (7.5\%) were children. The prevalence of LP was $57 \%$ in children and $66.7 \%$ in adults with an overall prevalence of $65.5 \%$, and the 11 years analysis of LP showed upwards trends. $74 \%$ of died children, $50 \%$ of discontinued children, $57 \%$ of transferred out children and $45 \%$ of children with immunological failure were delayed presenters for HIV care. Similarly, $64.7 \%$ of died adults, $65.3 \%$ of discontinued adults, $68.1 \%$ of transferred out adults and $78.7 \%$ of adults who had immunological failure presented late for the care. Factors for LP among adults were: being female, being married, having IF, having $\mathrm{Tb} / \mathrm{HIV}$ co-infection and having no history of HIV testing. No statistically significant predictor was found for LP among children.

Conclusions The prevalence LP was significant and majority HIV infected children and adults who presented late for HIV care had discontinued, transferred out and immunological failure. To address this, strategies such as unmanned aerial systems for transporting laboratory specimens, programs such as home and community-based HIV testing, 'opt out' and selftesting are compulsory.

\section{P3.90 HIV CARE CONTINUUM OUTCOMES IN ETHIOPIA: SURROGATES FOR UNAIDS 90-90-90 TARGETS FOR ENDING HIVIAIDS}

${ }^{1}$ Hailay Gesesew, ${ }^{1}$ Paul Ward, ${ }^{2}$ Kifle Hajito, 'Lillian Mwanri. ${ }^{1}$ Flinders University, Adelide, Australia; ${ }^{2}$ Jimma University, Jimma, Ethiopia

\subsection{6/sextrans-2017-053264.325}

Introduction How the UNAIDS 90-90-90 targets for ending HIV/AIDS by 2030 progressing have not yet been assessed in many countries including Ethiopia. We assessed HIV care continuum outcomes as a complex surrogate markers for the 9090-90 targets.

Methods Data were collected from 12 years anti-retroviral therapy (ART) records. For UNAIDS diagnosis target, early HIV diagnosis was considered as a surrogate marker. For treatment target, number of people on ART, number of people who discontinued from ART or transferred out, and number of people who had fair or poor adherence were used as surrogates. For viral suppression target, number of people with treatment success (combination of immunological and clinical) was used as a surrogate marker.

Results 8172 patients were enrolled for HIV cares in the period between 2003-2015. For the diagnosis target, 34.5\% patients knew their status early (43\%-children, 33\%-adults). For the treatment target, 65\% patients received ART, 1154 (21.9\%) patients discontinued from ART, 1015 (19.3\%) patients on ART transferred out to other sites, 916 (17\%) of patients on ART had fair or good adherence. For the virological suppression target, $80.7,80.3 \%$ and $65.8 \%$ of patients had immunological, clinical and treatment success displaying an estimated $66 \%$ of patients achieved the target.

Conclusion The finding reflects that an estimated $35 \%$ of patients knew their status timely, $65 \%$ of diagnosed patients received treatment and $66 \%$ of patients on ART achieved viral suppression. This is very far from the UNAIDS 90-90-90 targets underscoring the need for rigorous innovative methods such as unmanned aerial systems (or drones) for transporting laboratory specimens, immediate or same day ART initiation, community distribution of ART, runaway packs during conflict, and use of GenXpert for HIV viral load testing would significantly help to hit the target. Question: How resource constraint countries attain the 90-90-90 targets? 